\title{
Spatial quantum noise in singly resonant second-harmonic generation
}

\author{
P. Lodahl* \\ Optics and Fluid Dynamics Department, Risø National Laboratory, Postbox 49, DK-400o Roskilde, Denmark
} M. Saffman

Department of Physics, University of Wisconsin, 1150 University Avenue, Madison, Wisconsin 53706

Received July 23, 2001

\begin{abstract}
We study the spatial distribution of quantum noise in singly resonant second-harmonic generation. Calculations are performed below threshold for spatial modulational instability. For parameters for which the intracavity fields are modulationally stable the spatial spectrum shows maximum squeezing at $\mathbf{k}=0$, whereas under conditions of modulational instability we find maximum squeezing at finite wave number $|\mathbf{k}|=k_{c}$, where $k_{c}$ corresponds to the classical critical wave number. (c) 2002 Optical Society of America

OCIS codes: 270.0270, 270.6570, 190.0190, 190.4410.
\end{abstract}

$\chi^{(2)}$ frequency-conversion processes have proved to be efficient for generating quantum correlated light ${ }^{1}$ as well as complex spatial structures..$^{2-4}$ The synthesis of these two phenomena has led to the development of a new research field of spatial quantum structures. $^{5-8}$ Including spatial degrees of freedom, nonclassical phenomena such as squeezing, antibunching, and entanglement appear as functions of transverse coordinates. ${ }^{9,10}$ Our study of these states of light in the spatial domain is motivated by a basic interest in their quantum properties as well as in applications in the realm of low-noise image analysis and precision measurements. ${ }^{11-13}$

Parametric downconversion, in which a photon at $2 \omega$ is annihilated to create two photons at $\omega$, is the basic physical process that leads to nonclassical correlations in the outputs of the optical parametric oscillator and the optical parametric amplifier. A number of studies of the oscillator and the amplifier that included diffraction have revealed that signatures of the two-photon process are also present in the spatial structure of the field, so spatial squeezing, twin beams, and entanglement are all predicted to appear. ${ }^{5,6,10}$ The complementary process of secondharmonic generation (SHG), in which two photons at $\omega$ are annihilated to create one at $2 \omega$, is also known to produce nonclassical radiation fields. We demonstrate here that intracavity SHG also leads to spatial squeezing in both fundamental and harmonic outputs.

In this Letter we are concerned with singly resonant intracavity SHG. In this system the cavity is pumped by a coherent wave at fundamental frequency $\omega_{1}$, whereas the generated second harmonic at $\omega_{2}=2 \omega_{1}$ is nonresonant. In Ref. 14 it was shown that in the phase-mismatched configuration a transverse modulational instability exists that is the basis for a rich spectrum of spatial patterns. Although there is no threshold for the generation of the second harmonic (its strength increases with the intensity of the pump wave), a definite threshold exists for the onset of modulational instability. However, even below the instability threshold, precursors of the spatial patterns can be seen in the spatial distribution of noise. These below-threshold images have been studied in detail in the optical parametric oscillator (OPO) ${ }^{15}$ Here we analyze the below-threshold spatial distribution of quantum noise in singly resonant intracavity SHG.

The scaled quantized equations that describe singly resonant intracavity SHG are

$$
\begin{aligned}
\partial_{t} \hat{A}_{1}= & (-1+i \Delta) \hat{A}_{1}+f(\xi) \hat{A}_{1}^{\dagger} \hat{A}_{1}^{2}+i \nabla_{\perp}^{2} \hat{A}_{1} \\
& -g^{*}(\xi) \hat{A}_{1}^{\dagger}+\hat{A}_{2, \text { in }} \hat{A}_{, 1, \text { in }}, \\
\hat{A}_{2, \text { out }}= & g(\xi) \hat{A}_{1}^{2}+\hat{A}_{2, \text { in }} .
\end{aligned}
$$

Equations (1) are a generalization, including diffraction, of the two-photon-loss model studied previously in Refs. 16 and 17 and extended by Cabrillo et al.$^{18,19}$ to include phase mismatch. $\hat{A}_{1}$ and $\hat{A}_{2}$ are the annihilation operators for the fundamental and the second harmonic, respectively, with additional operators $\hat{A}_{1 \text {, in }}$ and $\hat{A}_{2, \text { in }}$ for the input fields. They satisfy the commutation relations $\left[\hat{A}_{j}(\mathbf{r}, t), \hat{A}_{j}^{\dagger}\left(\mathbf{r}^{\prime}, t^{\prime}\right)\right]=c_{j} \delta\left(\mathbf{r}-\mathbf{r}^{\prime}\right) \delta\left(t-t^{\prime}\right)$, etc., where $\mathbf{r}$ and $\mathbf{r}^{\prime}$ are transverse spatial coordinates and $c_{j}$ are constants that follow from the scalings of the spatial and temporal variables given in Ref. 14 . $\Delta$ is the scaled detuning of the fundamental, and the phase-mismatch of the SHG process is accounted for by the functions $f(\xi)=i 2 / \xi+[\exp (-i 2 \xi)-1] / \xi^{2}$ and $g(\xi)=[\exp (i 2 \xi)-1] / \xi$, where $\xi=\Delta k L, \Delta k$ is the phase mismatch, and $L$ is the length of the nonlinear crystal. The equations are given in the ideal situation in which the only cavity loss is the transmission of the input coupler.

The input-output relation ${ }^{20}$ for the fundamental cavity field expressed in the scaled amplitudes is $\hat{A}_{1, \text { out }}=2 \hat{A}_{1}-\hat{A}_{1, \text { in }}$. Assuming coherent and vacuum state inputs in the fundamental and the second harmonic, respectively, stationary classical solutions $A_{1}^{0}$ and $A_{2}^{0}$ of Eqs. (1) are obtained (see Ref. 14 for details). Within the semiclassical approximation ${ }^{21}$ we obtain linearized Langevin equations by substituting $\hat{A} \rightarrow A^{0}+b$ for all the operators in the equations. Fluctuating terms $b$ can be taken to be classical 
stochastic amplitudes, and input amplitudes $b_{1 \text {, in }}$ and $b_{2 \text {, in }}$ are delta correlated sources introduced to represent the quantum input noise. The semi-classical method can be used in the limit where the fluctuations are small compared with the classical mean values such that the following linearized equations are valid:

$$
\begin{aligned}
\partial_{t} b_{1}= & (-1+i \Delta) b_{1}+2 f(\xi)\left|A_{1}^{0}\right|^{2} b_{1}+f(\xi) A_{1}^{02} b_{1}^{*} \\
& +i \nabla_{\perp}^{2} b_{1}-g^{*}(\xi) A_{1}^{0 *} b_{2, \text { in }}+b_{1, \text { in }} \\
b_{1, \text { out }}= & 2 b_{1}-b_{1, \text { in }} \\
b_{2, \text { out }}= & 2 g(\xi) A_{1}^{0} b_{1}+b_{2, \text { in }} .
\end{aligned}
$$

Introducing the Fourier-transformed amplitudes $\beta_{j}(\mathbf{k}, \omega)=\int \mathrm{d} \mathbf{r} \int \mathrm{d} t b_{j}(\mathbf{r}, t) \exp [i(\mathbf{k} \cdot \mathbf{r}+\omega t)], j=1,2$, we can solved Eq. (2a) explicitly for Fourier components $\beta_{1}(\mathbf{k}, \omega)$ and $\beta_{1}^{*}(-\mathbf{k},-\omega)$. Substituting the solutions into Eqs. (2b) and (2c), we obtain the output Fourier components $\beta_{j \text {, out }}(\mathbf{k}, \omega)$. Using these solutions, we form the far-field output quadrature amplitudes:

$$
\begin{aligned}
P_{j, \text { out }}(\theta, \mathbf{k}, \omega)= & \beta_{j, \text { out }}(\mathbf{k}, \omega) \exp (-i \theta) \\
& +\beta_{j, \text { out }}^{*}(\mathbf{k},-\omega) \exp (i \theta),
\end{aligned}
$$

where $\theta$ specifies the quadrature of interest. The algebraic formulas are lengthy and are not given here.

The spectrum of output quadrature noise at a point in the far field characterized by $\mathbf{k}$ is defined as $S_{j}(\theta, \mathbf{k}, \omega)=\left\langle\left|P_{j, \text { out }}(\theta, \mathbf{k}, \omega)\right|^{2}\right\rangle /\left\langle\left|P_{j, \text { in }}(\theta, \mathbf{k}, \omega)\right|^{2}\right\rangle$. The spectrum can be written in the form $S_{j}(\theta, \mathbf{k}, \omega)=h_{1}(k)+\left[h_{2}(k) \cos (2 \theta)+h_{3}(k) \sin (2 \theta)\right] \delta(\mathbf{k})$, where $h_{j}(k)$ are lengthy expressions that depend on $k=|\mathbf{k}|$ and the cavity parameters. For $k>0$ these noise spectra are independent of the quadrature angle, and the noise is above the shot-noise level set by a coherent state of light, i.e., $S_{j}(\theta, \mathbf{k}, \omega) \geq 1{ }^{22}$ Hence individual points in the far field possess no quantum signatures. The same behavior is found in a quantum analysis of the OPO. ${ }^{23}$

However, by observing correlations between two conjugated points in the far field it is possible to beat the shot-noise level. For that purpose we introduce the quadratures $P_{j, \text { out }}^{+}(\theta, \mathbf{k}, \omega)=P_{j, \text { out }}(\theta, \mathbf{k}, \omega)+$ $P_{j, \text { out }}(\theta,-\mathbf{k}, \omega)$, which correspond to using a localoscillator field proportional to $\cos (\mathbf{k} \cdot \mathbf{r})$ in the near field. ${ }^{6}$ The corresponding spectrum is given by

$$
\begin{aligned}
& S_{j}^{+}(\theta, \mathbf{k}, \omega)=\frac{\left\langle\left|P_{j, \text { out }}^{+}(\theta, \mathbf{k}, \omega)\right|^{2}\right\rangle}{\left\langle\left|P_{j, \text { in }}^{+}(\theta, \mathbf{k}, \omega)\right|^{2}\right\rangle} \\
& =\frac{\left\langle\left|P_{j, \text { out }}(\theta, \mathbf{k}, \omega)\right|^{2}\right\rangle+\left\langle P_{j, \text { out }}(\theta, \mathbf{k}, \omega) P_{j, \text { out }}^{*}(\theta,-\mathbf{k}, \omega)\right\rangle}{\left\langle\left|P_{j, \text { in }}(\theta, \mathbf{k}, \omega)\right|^{2}\right\rangle},
\end{aligned}
$$

where we have used the fact that $\left|P_{j, \text { out } / \text { in }}(\theta, \mathbf{k}, \omega)\right|^{2}=$ $\left|P_{j, \text { out } / \text { in }}(\theta,-\mathbf{k}, \omega)\right|^{2} . \quad S_{j}^{+}(\theta, \mathbf{k}, \omega)$ is the spatial spectrum of squeezing ${ }^{9}$ that describes fluctuations obtained by addition of the quadratures measured at $\mathbf{k}$ and $-\mathbf{k}$ in the far field. The first term is just the noise from the individual directions; the second term accounts for the two-point correlations and reflects the two-photon nature of the $\chi^{(2)}$ process, as is well known, in particular from the OPO. When the second term dominates we can have $S_{j}^{+}(\theta, \mathbf{k}, \omega)<1$, which is a nonclassical effect.

First, we focus on the ideally phase-matched and resonant case $(\xi=\Delta=0)$ for which no spatial instability exists in the system. ${ }^{14}$ The spatial spectra of squeezing in this case are plotted in Fig. 1 as a function of transverse wave number $k$ for both the fundamental and the second harmonic. We note that in this case $A_{1}^{0}$ is real, so $S_{1}^{+}(\theta=0)$ and $S_{1}^{+}(\theta=\pi / 2)$ correspond to the amplitude and the phase quadratures, respectively. However, $A_{2}^{0}$ in this case is purely imaginary, it identifies $S_{2}^{+}(\theta=0)$ as the phase quadrature spectrum and $S_{2}^{+}(\theta=\pi / 2)$ as the amplitude quadrature spectrum. For $k=0$ we find optimum squeezing of $2 / 3$ in the fundamental (obtained for $A_{1}^{0}=1 / \sqrt{6}$ ), which reproduces the result of Paschotta et al. ${ }^{17}$ in the absence of diffraction. The maximum possible squeezing of $1 / 9$ in the second harmonic is obtained for $A_{1}^{0} \rightarrow \infty$. We see that the best squeezing is obtained for $k=0$, which is generally the case for parameters for which no spatial instability is found in the system. As $k$ increases to finite values, the degree of squeezing is reduced. The characteristic spatial bandwidth reflects the relative role of diffraction. Using the scalings given in Ref. 14, we have that $\tilde{k}=k \sqrt{2 \gamma k_{0} n^{2} / c}$, where $\tilde{k}$ is the value of $k$ in physical units, $\gamma$ is the cavity decay rate, $k_{0}$ is the vacuum wave number, $n$ is the index of refraction, and $c$ is the speed of light. Thus, as decay rate $\gamma$ increases, the relative role of diffraction is reduced and the spatial bandwidth of the squeezing gets wider.

For other system parameters a spatial instability exists at a finite transverse wave number. For these parameters the best squeezing is achieved at a transverse wave number that approaches the critical instability wave number predicted by linear stability analysis. ${ }^{14}$ As is shown in Fig. 2, increasing the pump toward threshold improves the squeezing at a transverse wave

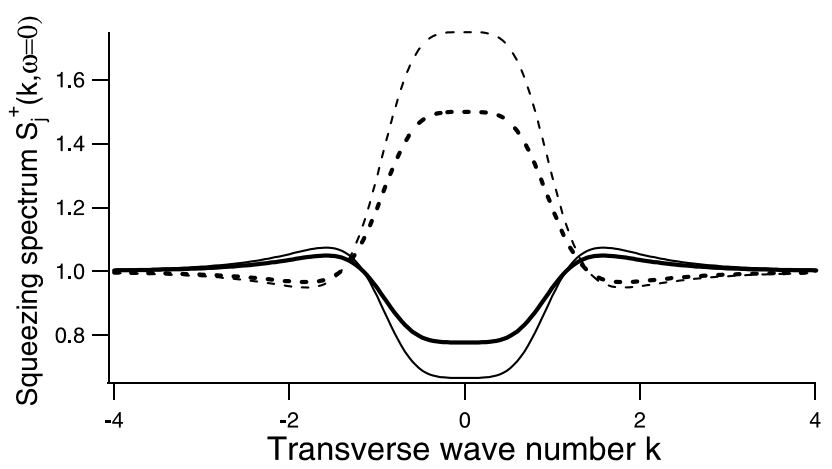

Fig. 1. Squeezing spectra for the amplitude (solid curves) and the phase (dashed curves) quadratures as a function of transverse wave number for $\Delta=\xi=\omega=0$ and $A_{1}^{0}=1 / \sqrt{6}$. The thinner and thicker curves are the fundamental and the second harmonic, respectively. 


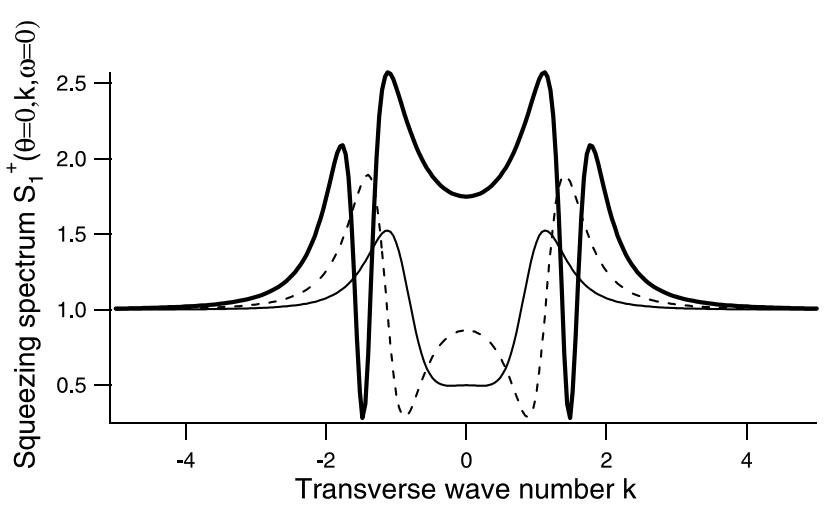

Fig. 2. Squeezing spectra for the $S_{1}^{+}(\theta=0)$ quadrature for $\Delta=0, \xi=5, \omega=0$, and $\left|A_{1}^{0}\right|^{2}=0.6$ (thinner curve), $\left|A_{1}^{0}\right|^{2}=$ 1.3 , (dashed curve), and $\left|A_{1}^{0}\right|^{2}=2.5$ (bolder solid curve). The threshold for spatial instability sets in at $\left|A_{1}^{0}\right|^{2}=3.56$.

number that approaches the critical value $k_{c}=$ $\sqrt{\Delta+\Delta^{\mathrm{NL}}}$, which is 1.73 for the parameters used in the figure.

This behavior is closely related to that found by Lugiato and Gatti ${ }^{6}$ in the OPO. They showed there that maximum squeezing occurs at $k=0$ in the absence of modulational instability and at $k=k_{c}$ when there is modulational instability. Significant differences between the OPO and SHG are that for the OPO the oscillation threshold coincides with the threshold for transverse instability such that $\Delta^{\mathrm{NL}}=0$ and $k_{c}=\sqrt{\Delta}$ and that below the threshold for modulational instability the squeezing manifests itself as squeezed vacuum fluctuations. In SHG there is always a finite output field, so $\Delta^{\mathrm{NL}}=2 \operatorname{Im}(f) \mid A_{1}$, threshold $\left.\right|^{2}$ is not zero, and we obtain spatial squeezing of a bright field with a coherent amplitude. In addition, whereas in the OPO the squeezing becomes asymptotically perfect at $k=k_{c}$, such is not the case in SHG.

Finally, we note that although the results given here predict the generation of spatial squeezing in intracavity second-harmonic generation, Eq. (1a) describes a broader range of optical interactions. When phase mismatch $\xi$ is an integer multiple of $\pi$, the real part of function $f$ vanishes and $g=0$ so Eq. (1a) then coincides with the quantized version of the Lugiato-Lefever model ${ }^{24}$ for a Kerr medium in a cavity. The results given in Fig. 1 therefore describe the squeezing spectra for a Kerr nonlinearity in an optical cavity. Furthermore, as was shown in Ref. 5, the quantized version of the Lugiato-Lefever model implies nonclassical twin-beam intensity correlations between the beams emitted off axis under conditions of spatial instability. Although such is not shown explicitly here, we suspect that similar twin-beam correlations are also present for a wider range of phase-mismatch values in SHG.
Support from the European Science Foundation PHASE network is acknowledged. M. Saffman is an A. P. Sloan Foundation fellow; his e-mail address is msaffman@facstaff.wisc.edu.

*Present address, Norman Bridge Laboratory of Physics 12-33, California Institute of Technology, Pasadena, California 91125.

\section{References}

1. H. J. Kimble, Phys. Rep. 219, 227 (1992).

2. G.-L. Oppo, M. Brambilla, and L. A. Lugiato, Phys. Rev. A 49, 2028 (1994).

3. M. Vaupel, A. Maître, and C. Fabre, Phys. Rev. Lett. 83, 5278 (1999).

4. R. A. Fuerst, D.-M. Baboiu, B. Lawrence, W. E. Torruellas, G. I. Stegeman, S. Trillo, and S. Wabnitz, Phys. Rev. Lett. 78, 2756 (1997).

5. L. A. Lugiato and F. Castelli, Phys. Rev. Lett. 68, 3284 (1992).

6. L. A. Lugiato and A. Gatti, Phys. Rev. Lett. 70, 3868 (1993).

7. G. Grynberg and L. A. Lugiato, Opt. Commun. 101, 69 (1993).

8. E. M. Nagasako, R. W. Boyd, and G. S. Agarwal, Phys. Rev. A 55, 1412 (1997).

9. M. I. Kolobov, Rev. Mod. Phys. 71, 1539 (1999).

10. A. Gatti, E. Brambilla, L. A. Lugiato, and M. I. Kolobov, Phys. Rev. Lett. 83, 1763 (1999).

11. S.-K. Choi, M. Vasilyev, and P. Kumar, Phys. Rev. Lett. 83, 1938 (1999).

12. S.-K. Choi, M. Vasilyev, and P. Kumar, Phys. Rev. Lett. 84, 1361 (2000), erratum of Ref. 11.

13. C. Fabre, J. B. Fouet, and A. Maître, Opt. Lett. 25, 76 (2000).

14. P. Lodahl and M. Saffman, Phys. Rev. A 60, 3251 (1999).

15. A. Gatti and L. Lugiato, Phys. Rev. A 52, 1675 (1995).

16. M. J. Collett and R. B. Levien, Phys. Rev. A 43, 5068 (1991).

17. R. Paschotta, M. Collett, P. Kürz, K. Fiedler, H.-A. Bachor, and J. Mlynek, Phys. Rev. Lett. 72, 3807 (1994).

18. C. Cabrillo, J. L. Roldán, and P. García-Fernández, J. Opt. Soc. Am. B 17, 440 (2000).

19. Note that the model studied in Ref. 17 did not include the effect of phase mismatch on the second harmonic, which is described by a factor of $g$ in Eq. (1b).

20. C. W. Gardiner and M. J. Collett, Phys. Rev. A 31, 3761 (1985).

21. S. Reynaud and A. Heidmann, Opt. Commun. 71, 209 (1989).

22. The abrupt transition between $k=0$ and $k$ finite as a result of the delta functions is an artifact of the assumption of an infinitely extended plane-wave pump. Allowing for a pump beam of finite extent would ease the transition.

23. J. Jeffers and G.-L. Oppo, Phys. Rev. A 60, 2393 (1999).

24. L. A. Lugiato and R. Lefever, Phys. Rev. Lett. 58, 2209 (1987). 